\title{
Setting Up the Perimeter of Tolerance: Insights into mTEC Physiology
}

\author{
Rodrigues PM1, Peterson P2, Alves NL3.
}

1-Instituto de Investigação e Inovação em Saúde, Universidade do Porto, Porto, Portugal; Thymus Development and Function Laboratory, Instituto de Biologia Molecular e Celular, Porto, Portugal. 2-Molecular Pathology, Institute of Biomedicine and Translational Medicine, University of Tartu, Tartu, Estonia.

3-Instituto de Investigação e Inovação em Saúde, Universidade do Porto, Porto, Portugal; Thymus Development and Function Laboratory, Instituto de Biologia Molecular e Celular, Porto, Portugal.

*Correspondence: nalves@ibmc.up.pt (N.L. Alves).

Originally published in Trends in immunology, 39(1):2-5, November, 24, 2017.

Doi: $10.1016 /$ j.it.2017.11.001

Medullary thymic epithelial cells (mTECs) play a central role in T cell tolerance. However, how the mTEC compartment is maintained remains elusive. We review recent discoveries on new transcription factors involved in MTEC homeostasis and discuss the possibility that their actions might be facilitated by the unique biology of mTECs.

The generation of multifunctional and self-tolerant $\mathrm{CD} 4^{+}$and $\mathrm{CD} 8^{+} \alpha \beta$ T cells proceeds sequentially within dedicated microenvironments of the thymus formed by cortical TECs (cTECs) and mTECs [1]. Despite deriving from common bipotent progenitors, cTECs and mTECs specialize into functionally distinct cell types [2]. While cTECs drive T cell lineage commitment and positive selection, mTECs mediate the deletion of autoreactive thymocytes or promote their clonal deviation into regulatory T cells [1]. The central role of mTECs in tolerance induction depends on their unique capacity to express virtually all self-antigens, including tissue-restricted antigens (TRAs). This so-called promiscuous gene expression (pGE) potential is acquired during mTEC maturation in part through the action of two transcriptional regulators, Autoimmune regulator (Aire) and Fezf2, that coordinate the expression of a broad range of TRAs [3]. The development of mTECs commences during organogenesis and continues during adulthood. In recent years the identification of distinct subtypes of mTEC precursors, such as Claudin3,4+stage-specific embryonic antigen (SSEA) ${ }^{+}$, Claudin $3,4^{+}$, receptor activator of NF- $\mathrm{\kappa B}(\mathrm{RANK})^{+}$, and podoplanin ${ }^{+}$ cells, has emphasized the dynamics of this process [4]. NF- $\kappa \mathrm{B}$ signaling is critical to mTEC differentiation and is triggered by the engagement of members of the TNF receptor superfamily (TNFRSF) - RANK, CD40, and LT $\beta R$ - by their ligands expressed in developing thymocytes [1]. Despite substantial advances in our understanding of the blueprint of mTEC differentiation, the molecular mechanisms that control the maintenance of the thymic medulla in postnatal life remain poorly defined. Interestingly, mature mTECs turnover every 1-2 weeks, indicating the need for their regular replacement by upstream precursors. Given that genetic or age-related defects in mTECs are linked to the development of autoimmunity, the study of how these cells influence thymic activity is of fundamental and medical importance. In this Forum we summarize recent studies that have uncovered the role of new classes of hitherto overlooked transcription factors (TFs) in the selective regulation of mTEC homeostasis.

Our laboratories independently generated experimental evidence using conditional loss-offunction mouse models that adds novel pieces to our understanding of thymus medulla 
homeostasis and function. Rodrigues et al. analyzed mice with a disrupted Trp53 gene in TECs and showed that p53 is critical for mTEC maintenance and function [5]. TEC-intrinsic p53 deficiency causes a specific reduction in the size of the mTEC compartment, decreasing the expression of RANK in mTECs and conditioning their responsiveness to RANK stimulation. Beyond the functional link to RANK, we found that 553 regulates a broad transcriptional program in mTECs, including the expression of TRAs and genes associated with core processes of mTEC biology [5]. However, it is presently unclear how p53 maintains the mTEC niche. Given that p53 controls a basal transcriptional program in unstressed cells [6], the reduced mTEC compartment in p53 conditional knockout (KO) mice might result from broad failures in genes linked to essential mTEC biology. In a complementary study, Haljasorg et al. generated TEC-specific IFN regulatory factor 4 (IRF4) KO mice and found that disruption of Irf4 leads to an increase in the frequency of mature mTECs and alters the expression of important chemokine and costimulatory molecules in these cells [7]. This observation raises the possibility that IRF4 controls a novel checkpoint in mTEC differentiation. Intriguingly, the absence of p53 and IRF4 provokes a common reduction in thymic regulatory $\mathrm{T}$ cell numbers, leading to peripheral autoimmune manifestations [5, 7]. Future studies should dissect to what extent p53 and IRF4 control distinct and/or overlapping programs in mTEC biology. Interestingly, both reports suggest the involvement of TNFRSF signaling in the regulation of the expression of p53 and IRF4. While p53 is induced by RANK and CD40 stimulation [5], IRF4 expression is specifically controlled by RANK activation [7]. The RANK-IRF axis was previously implicated in mTEC differentiation with the observation that the activation of IRF7 downstream of RANK controls the production of IFN- $\beta$, which in turns promotes mTEC development [8]. Thus, p53-, IRF4-, and IRF7-driven pathways seem to branch downstream of TNFRSF-induced activation of the NF- $\kappa B$ signaling pathway and coordinate multiple aspects of mTEC homeostasis. However, it remains to be further investigated whether these pathways can be activated by alternative signals (Figure 1).

Besides agonist signals, the TNFRSF-mediated pathway is tempered by negative regulators that control the bioavailability of TNFRSF ligands, the expression of the receptors, and the intensity of downstream signaling. Osteoprotegerin (OPG) is one of these regulators, operating as a soluble decoy receptor for RANK ligand. The expression of OPG in mTECs is controlled by the TF Spi-B, which is in turn activated by RANK [9]. Hauri-Hohl et al. identified TGF- $\beta$ as an additional negative regulator of the mTEC compartment. Genetic ablation of TGF- $\beta$ signaling in TECs expanded the mTEC niche and the functional capacity of the thymus medulla to maintain tolerance induction. Mechanistically, TGF- $\beta$ signaling dampens the NF- $\kappa$ B pathway elicited by RANK and CD40 engagement [10]. These findings suggest that OPG and TGF- $\beta$ are important liaisons in the negative feedback mechanism controlling TNFRSF signaling during mTEC differentiation.

Interestingly, two recent reports demonstrated that alternative signaling pathways also participate in the maintenance of the medullary microenvironment in the adult thymus. Satoh et al. and Lomada et al. have shown that conditional inactivation of Stat3 in TECs prevents the expansion of thymic medullary areas during postnatal life $[11,12]$. Despite exhibiting a reduction in the mTEC compartment similar to that in p53 conditional $\mathrm{KO}$ mice, the number of thymic regulatory $\mathrm{T}$ cells is unaltered in mice with STAT3-deficient TECs, indicating that STAT3 and p53 regulate complementary programs in mTECs. STAT3 is activated by various cytokines and growth factors in vivo. Satoh et al. provide further evidence that STAT3 signaling in TECs is induced by the epithelial growth factor receptor (EGFR), although the intrathymic physiological source of its ligand remains unclear [11]. Cosway et al. have recently shown that targeted deletion of LT $\beta$ R in TECs disturbs the architecture of mTECs without an overt effect on their functionality [13]. Unlike the profound defects caused by the lack of Relb signaling [14], conditional deficiency of LT $\beta$ R does not affect thymic regulatory $\mathrm{T}$ cells and induces a milder phenotype reminiscent of STAT3 deficiency. The new studies highlight that the establishment, maintenance, and architecture of the 
thymus medulla are determined by the integration of multiple signals, although it remains unclear whether proper mTEC structure is required for its functionality. Further studies should aim to unravel the genetic program driven by the recently identified TFs in mTECs and their possible relationship in maintaining a functional mTEC compartment (Figure 1).

Intriguingly, the specific role of these TFs in mTEC biology was demonstrated in experimental models in which their genes were targeted in both cTECs and mTECs. On the one hand, the enriched expression and/or activation of these proteins in mTECs might determine their specific influence. On the other hand, we hypothesized that the unique transcriptional and epigenetic states of mTECs [3] can facilitate the access of these TFs to their targets. According to this conjecture, a given TF drafts behind the molecular machinery involved in regulating pGE expression and exploits the induced changes in the chromatin to initiate a specific genetic program in mTECs. However, the action of the newly identified TFs in MTEC homeostasis seems to be distinct from the prototypical roles of NF- $\kappa B$ in mTEC maturation or Aire and Fezf2 in TRA expression. Instead, these novel pathways appear to operate as a key rheostat to sustain the regular activity of the thymus medulla under physiological conditions, preventing the emergence of autoimmunity. Ultimately, understanding the mechanisms controlling the limits of the medullary microenvironment might lead to a better comprehension of the rules balancing immunity and tolerance induction.

\section{ACKNOWLEDGMENTS}

The European Research Council (ERC) under the EU's Horizon 2020 research and innovation program (grant agreement No 637843 - TEC_Pro) - starting grant attributed to N.L.A - supports the studies from the laboratory of N.L.A. The studies from the laboratory of P.P. are supported by Estonian Research Agency grant IUT2-2. The authors thank the members of their laboratories for their past and present contributions. They apologize for not referring to all primary literature owing to space limitations

\section{REFERENCES AND NOTES}

1. Anderson, G. and Takahama, Y. (2012) Thymic epithelial cells: working class heroes for T cell development and repertoire selection. Trends Immunol. 33, 256-263

2. Alves, N.L. et al. (2014) Serial progression of cortical and medullary thymic epithelial microenvironments. Eur. J. Immunol. 44, 16-22

3. Takaba, H. and Takayanagi, H. (2017) The mechanisms of T cell selection in the thymus. Trends Immunol. 38, 805-816

4. Alves, N.L. and Ribeiro, A.R. (2016) Thymus medulla under construction: time and space oddities. Eur. J. Immunol. 46, 829-833

5. Rodrigues, P.M. et al. (2017) Thymic epithelial cells require p53 to support their long-term function in thymopoiesis in mice. Blood 130, 478-488

6. Munoz-Fontela, C. et al. (2016) Emerging roles of p53 and other tumour-suppressor genes in immune regulation. Nat. Rev. Immunol. 16, 741-750

7. Haljasorg, U. et al. (2017) Irf4 expression in thymic epithelium is critical for thymic regulatory T cell homeostasis. J. Immunol. 198, 1952-1960

8. Otero, D.C. et al. (2013) IRF7-dependent IFN-b production in response to RANKL promotes medullary thymic epithelial cell development. J. Immunol. 190, 3289-3298

9. Akiyama, N. et al. (2014) Limitation of immune toleranceinducing thymic epithelial cell development by Spi-B-mediated negative feedback regulation. J. Exp. Med. 211, 2425-2438 
10. Hauri-Hohl, M. et al. (2014) A regulatory role for TGF-b signaling in the establishment and function of the thymic medulla. Nat. Immunol. 15, 554-561

11. Satoh, R. et al. (2016) Requirement of Stat3 signaling in the postnatal development of thymic medullary epithelial cells. PLoS Genet. 12, e1005776

12. Lomada, D. et al. (2016) Stat3 signaling promotes survival and maintenance of medullary thymic epithelial cells. PLoS Genet. 12, e1005777

13. Cosway, E.J. et al. (2017) Redefining thymus medulla specialization for central tolerance. J. Exp. Med. 214, 3183-3195

14. Cowan, J.E. et al. (2013) The thymic medulla is required for Foxp3+ regulatory but not conventional CD4+ thymocyte development. J. Exp. Med. 210, 675-681 
(A)

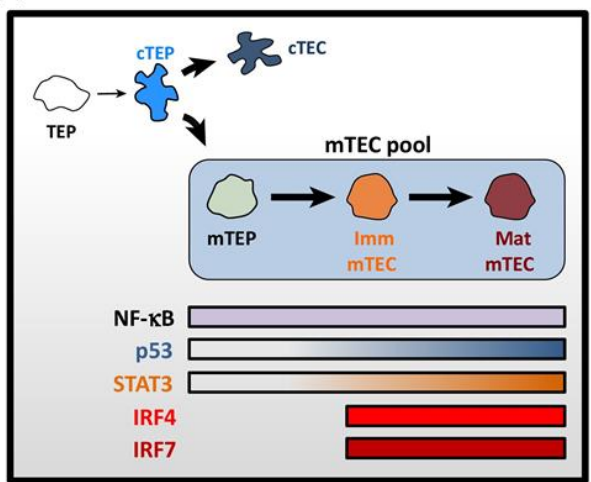

(B)

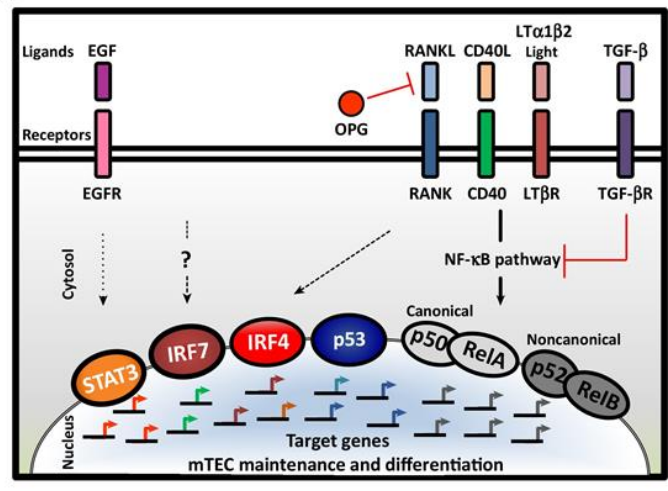

Trends in Immunology

Figure 1 - Proposed Model for the Action of the Recently Identified Regulators of Medullary Thymic Epithelial Cell (mTEC) Homeostasis. (A) TEC progenitors (TEPs) traverse through a 'transitional TEC progenitor' stage that expresses phenotypic and molecular traits associated with cortical TEC (cTEC) precursors (cTEPs) before the commitment to a cTEC or mTEC fate [2]. The NF-кB pathway regulates various stages of their differentiation, from mTEC progenitors (mTEPs) to immature (Imm) and mature (Mat) mTECs [4]. While p53 [5] and STAT3 [11,12] appear to control the maintenance of the broad mTEC compartment, IFN regulatory factor (IRF) 4 [7] and IRF7 [8] seem to regulate the immature-mature mTEC transition. (B) Molecular interplay between the distinct signaling pathways and transcription factors (TFs) governing the homeostasis and differentiation of mTECs. The right side represents the well-recognized role in mTECs of members of the TNF receptor superfamily (TNFRSF) - receptor activator of NF- $\mathrm{BB}$ (RANK), CD40, and LT $\beta \mathrm{R}$ - which on interaction with their respective ligands activate the canonical and noncanonical NF-KB pathway. This pathway of mTEC differentiation is regulated at multiple levels. Osteoprotegerin (OPG) acts as a decoy receptor for RANK ligand (RANKL), inhibiting RANK signaling [9]. Additionally, signaling through the TGF- $\beta$ receptor (TGF- $\beta$ R) negatively regulates the NF- $\mathrm{KB}$ pathway [10]. The left side represents the role of p53-, IRF4-, IRF7-, and STAT3-mediated signaling in the coordination of mTEC differentiation and homeostasis. While p53 [5], IRF-4 [7], and IRF7 [8] appear to be activated downstream of TNFRSF signaling, STAT3 $[11,12]$ is suggested to be engaged by signaling through the epithelial growth factor receptor (EGFR). It remains unclear whether these pathways can be activated by other, asyet-undefined signals (represented by '?'). Another uncertainty pertains to the overlapping or distinct nature of the gene expression program driven by the recently identified TFs in mTECs. Black lines with superscript colored arrows represent purported target genes. 